\title{
Analysis and Assessment of State Relevance in HMM-Based Feature Extraction Method
}

\author{
Rok Gajšek, Simon Dobrišek, and France Mihelič \\ Faculty of Electrical Engineering, University of Ljubljana, \\ Tržaška 25, SI-1000 Ljubljana, Slovenia \\ \{rok.gajsek, simon.dobrisek, france.mihelic\}@fe.uni-lj.si \\ http://luks.fe.uni-lj.si/
}

\begin{abstract}
In the article we evaluate the importance of different HMM states in an HMM-based feature extraction method used to model paralinguistic information. Specifically, we evaluate the distribution of the paralinguistic information across different states of the HMM in two different classification tasks: emotion recognition and alcoholization detection. In the task of recognizing emotions we found that the majority of emotion-related information is incorporated in the first and third state of a 3-state HMM. Surprisingly, in the alcoholization detection task we observed a somewhat equal distribution of task-specific information across all three states, resulting in constantly producing better results if more states are utilized.
\end{abstract}

Keywords: emotion recognition, HMM, HMM adaptation, MAP.

\section{Introduction}

Modern systems for paralinguistic information analysis from speech such as, speaker's gender or age, emotion or other psychophysical effects, are mostly based on a procedure of Universal Background Model (UBM) adaptation [1 2]. Usually the UBM is represented as a Gaussian Mixture Model (GMM) and the adaptation is done following the maximum a posteriori (MAP) adaptation criteria. In our previous work [3] we presented a similar adaptation scheme where instead of the GMM, an HMM likelihood function is used as an UBM. The adaptation criteria remains MAP. We have shown that through the use of an HMM, whose components represent particular phones, the paralinguistic information can be better represented in the values of the adapted means of Gaussian distributions. In this paper we present an evaluation and assessment of the distribution of the paralinguistic information across the states of the HMM. Specifically, we evaluate the recognition accuracy of systems based on the mean vectors from different combinations of HMM states.

In order to reliably assess the importance of different HMM states for paralinguistic recognition tasks we selected two corpora with different types of speech. For the emotion recognition evaluation we used the FAU Aibo Emotion Corpus (FAU-Aibo) [4] which consists of spontaneous speech uttered by German children. For the second task of alcohol intoxication detection a Slovenian corpus of alcoholized speech is used [5].

The reminder of the paper is organized as follows. In Section 2 a description of FAUAibo and Vindat databases is given. Section 3 provides an overview of the HMM-based 
feature extraction method, proposed for the various tasks of paralinguistic information recognition, as well as our reasoning for evaluating the impact of using different combinations of HMM states. Experiments and results are discussed in Section 4 and in Section 5 our final conclusions are given.

\section{Databases}

FAU-Aibo corpus [4 6] contains spontaneous children's speech labeled according to the emotional states being expressed in the recordings. The Wizard-of-Oz type of scenario was designed, where children utter commands to the little robot dog Aibo, while a person in the back is actually controlling the motion of the robot. This setup is effective for inducing an emotional response from the participant since the controller can intentionally disobey the participant's commands. In order to have the same behavior of the robot for all the participants, its movement and reactions to certain tasks were predefined. The session included five object localization tasks with children directing the robot to the defined spot, and the main task of leading the robot through a predefined path and at certain stops commanding the robot to perform a particular action. On one hand, the predefined and disobedient actions of the robot dog lead to frustration and other negative emotions, while the actions, compliant with the children's commands, induce positive emotions.

The FAU-Aibo database contains the recordings of 51 children between the ages of 10 and 13, of which 30 were female and 21 were male. In our experiments we follow a 2-class protocol of the Interspeech 2009 Emotion Challenge described in [1]. The class of negative emotions (NEG) consists of emotional labels angry, touchy, reprimanding and emphatic, while the idle class (IDL) consists of all non-negative emotional states. The sessions took place at two schools and according to the location of the recording the corpus is split into the training (school \#1) and test (school \#2) sets. The number of utterances per class is shown in Table [1] where an imbalance towards the idle class (IDL) can be observed. The number of samples for the negative class (NEG) is approximately half of the number of samples for the IDL class, which is usually the case in databases of spontaneous emotions.

Table 1. Number and distribution of samples in (a) the FAU-Aibo and in (b) the Vindat

(a) FAU-AIBO 2-class: negative vs. idle

\begin{tabular}{c||c|c|c}
\hline$\#$ & IDL & NEG & $\sum$ \\
\hline Train & 6601 & 3358 & 9959 \\
Test & 5792 & 2465 & 8257 \\
\hline$\sum$ & 12393 & 5823 & $\mathbf{1 8 2 1 6}$
\end{tabular}

(b) VINDAT 2-class: non-alcoholized vs. alcoholized

\begin{tabular}{c||c|c|c}
\hline$\#$ & NON-ALCO & ALCO & $\sum$ \\
\hline All & 450 & 421 & $\mathbf{8 7 1}$
\end{tabular}




\subsection{VINDAT}

The VINDAT database [5] contains the recordings of people speaking at different levels of alcohol intoxication. Ten Slovene speakers, five men and five women, took part in the recording sessions. The average age of the adults was 35 years. The recording session for each speaker consisted of two parts. For the first part, fourteen Slovenian words were selected based on their demanding pronunciation (for example, the Slovene translation of otorhynolaryngologist - "otorinolaringologinja"). The selected words formed the center of a sentence, with meaningful words added left and right, in order to avoid the changes in speed and intonation that are usually found at the beginning and the end of spoken utterances. In the second part, the speakers repeated sentences previously read to them by the operator in an attempt to record speech closer to the natural speaking style.

The participants were recorded in three sessions based on the amount of the consumed alcohol. The alcohol levels were measured by a hand-held indicator, usually employed by the police for the inspection of drivers on the roads. The device measures the level of intoxication as the amount of milligrams per liter of exhaled air $(\% 0)$. In the first session, the participants were sober with $0 \%$. Before the second and the third session, each speaker consumed a selected amount of alcoholic beverage and measured the level of alcoholization prior to recording session. Understandably, at least 15 minutes passed since the last drink was consumed, before each measurement was made in accordance with the instructions of the alcohol-level indicator. Half of the participants exhibited $0.5 \%$ (which is a legal limit for driving in Slovenia) prior to the second recording, and by the last session, all participants had an alcohol level above $0.5 \%$.

For our task of alcoholization recognition, all the utterances labeled as less than $0.5 \%$ were assigned to the non-alcoholized class (NON-ALCO) and the rest (equal or more than $0.5 \%$ ) were assigned to the alcoholized class (ALCO). The threshold was set in accordance with the local legal limit for driving, as well as in accordance with the Intoxication Sub-challenge in [2] and the comparative database of [7]. In the lower part of Table 11 the distribution among the NON-ALCO and the ALCO classes is presented and it can be seen that the classes are almost balanced. While the text uttered in the database recordings was predefined and should as such match the content of the recordings, after listening to all the utterances, approximately $10 \%$ were not in accordance with the proposed text. These exceptions were carefully corrected enabling the use of transcriptions in our HMM adaptation.

\section{HMM-Based Feature Extraction Method}

HMM-based feature extraction method [3] is a modification of the standard GMMUBM adaptation [8]. In the classification scheme based on GMM-UBM adaptation, the first step is to build the UBM model using all available training data. Once the UBM is acquired a set of adapted GMMs is estimated based on the UBM and each particular sample in the corpus. From the set of newly adapted models the means of Gaussian distributions are extracted separately for each model and transformed into a vector, sometimes referred to as a super-vector. The set of super-vectors is then used 
in the selected classifier. In [3] we proposed to use an HMM model instead of a GMM to represent the UBM. The idea is based on the fact that the components in the GMM are constructed based on statistical information whereas for the monophone HMM the components represent individual allophone. Hence, the super-vector's elements, which are derived from the adaptation procedure, represent utterance specific information seperatly for each allophone contained in the utterance. The elements that correspond to the phonemes missing in the specific utterance are not updated and keep the value from the UBM. The procedure is described in detail below.

The adaptation formula for the mean vector of the mixture component $i$ in the HMM state $s$ is

$$
\hat{\boldsymbol{\mu}}_{s i}=\alpha_{s i} \mathbf{E}_{s i}(X)+\left(1-\alpha_{s i}\right) \boldsymbol{\mu}_{s i}^{U B M},
$$

where $\mu_{s i}^{U B M}$ is the mean of the HMM-UBM $s$ state and $i$-th mixture, $\mathbf{E}_{s i}(x)$ is the mean of the observed adaptation data and $\alpha_{s i}$ is the adaptation parameter. Similarly to the case of GMM adaptation, it is defined as

$$
\alpha_{s i}=\frac{n_{s i}}{n_{s i}+\tau},
$$

where $\tau$ is the relevance factor and $n_{s i}$ is the occupation likelihood of the adaptation data, defined as

$$
n_{s i}=\sum_{t=1}^{T} \operatorname{Pr}_{s i}(t) \text {. }
$$

The mean of the observed adaptation data $\mathbf{E}_{s i}(x)$ from Eq. 11 is calculated as

$$
\mathbf{E}_{s i}(X)=\frac{\sum_{t=1}^{T} \operatorname{Pr}_{s i}(t) \mathbf{x}_{t}}{n_{s i}},
$$

where $\operatorname{Pr}_{s i}$ denotes the probability of occupying the mixture $i$ of state $s$ at time $t$, and $\mathbf{x}_{t}$ is the adaptation data feature vector at time $t$. One can see that the MAP adaptation of the means of the HMM (Eq. (14)) is an extension of the MAP adaptation of the GMM, hence, if there is only one state in the HMM, the equations become identical.

The adaptation formula for means (Eq. (11) is made up of two terms, the UBM value of mean $\mu_{s i}^{U B M}$ and the calculated mean of the observed adaptation data $\overline{\boldsymbol{\mu}}_{s i}$, both weighted by the combination of the occupation likelihood $n_{s i}$ and the relevance factor $\tau$. The distance between the new MAP estimated mean and the UBM initial mean is determined by the occupation likelihood $n_{s i}$ for the particular component $i$ of state $s$. The higher the value of $n_{s i}$, the more influence the adaptation data has on the new adapted mean. And vise-versa, if the value of the $n_{s i}$ for the $i$ component is small, the value of the mean vector will remain similar to the initial UBM mean. Likewise, the relevance factor $\tau$ controls the weighting of the prior mean and the influence of the adaptation data as well. However, it is independent of the adaptation data, and thus controls mostly the speed of the convergence. It should be noted that equations 1,3] are run iteratively until either the predefined number of iterations is reached, or the difference between the new and the old means is smaller than a predefined threshold. Thus, the notation $\boldsymbol{\mu}_{s i}^{U B M}$ is appropriate only for the first iteration, when the value of 
the mean vector from the UBM is used, and should be, in later iterations, better denoted by $\mu_{s i}$, symbolizing the current value of the mean being updated. However, the symbol $\boldsymbol{\mu}_{s i}^{U B M}$ is left in the equations since it better represents the idea of adapting the same HMM-UBM for each particular utterance.

Although the MAP adaptation can be used to update all the parameters of the HMMUBM, we leave the weights, covariances, transition probabilities and state probabilities intact. Hence, all utterance-specific information or differences from the "general" speech, represented by the HMM-UBM, is captured in the new vectors of means. In this way, the exploitation of the means as a classification feature is enabled.

Once the above-described adaptation of the HMM-UBM for all train and test samples is finished, a set of new HMMs is obtained. These HMMs share the same values for all the parameters, except for the means of the Gaussian densities in the states. Next, for every utterance-specific HMM, the mean vectors are pooled together and combined in a new super-vector. The size of the super-vector is the number of Gaussians in the HMM-UBM times the dimension of the front-end acoustic feature vector. The set of super-vectors is obtained for the training and the test sets. These sets represent a suitable input to the classification, which is in our case realized by the SVM classification, like in the case of the GMM adaptation. Hence, the improvement of our HMM-UBM-MAP against the GMM-UBM-MAP modeling can be easily demonstrated.

\subsection{Analysis of the HMM States}

The HMM-based feature extraction method, described in the previous section, updates means in all 3 states of the HMM according to the MAP criteria. We want to evaluate if the paralinguistic information is equally distributed across all three states. In the case of stationary allophones the central state represents the segment of the phone were the speech signal exhibits as quasi-stationary, and the first and third state capture transitions to and from the allophone. Therefore, we devised an experimental protocol where would compare using only the means form the central states of HMM, only the means from the first and the third state against using means from all three states. This way we could reliable assess the importance of particular states to the information about the speaker's emotional state or intoxication level.

\section{Experiments and Results}

Both systems, for emotion recognition and intoxication detection, were based on MFCCs $0-12$ and their $\Delta s$, calculated on 20 millisecond frames, with a delay of 10 milliseconds between the frames. The filter bank consisted of 22 Mel-spaced filters. The 0 -th cepstral coefficient corresponds to the energy of the frame.

The UBM training and the adaptation process was done using the HTK toolkit [9] with the MAP relevance factor $\tau$ set to 16 and the number of the MAP iterations limited to 5. After the set of super-vectors was constructed from the means of the adapted GMMs a SVM classification was employed. A linear kernel was trained using sequential minimal optimization algorithm (SMO) was used for the training of the 
Table 2. Comparison of emotion recognition performance using different combinations of HMM states for the FAU-Aibo corpus

\begin{tabular}{c||c|c|c}
\multirow{2}{*}{\multicolumn{1}{c||}{ \# Gaussians }} & \multicolumn{3}{c}{ Number of used HMM states } \\
\cline { 2 - 4 } & States 1,2 and 3 & State 2 & States 1 and 3 \\
\hline 120 & $70.3 \%$ & $69.3 \%$ & $70.6 \%$ \\
200 & $69.2 \%$ & $68 \%$ & $69.5 \%$ \\
\hline
\end{tabular}

SVM classifier as implemented in the Weka toolkit [10]. The same SVM classification parameters were used in all the tests.

In emotion recognition experiments, the total number of allophones used was 40 . Consequently, the total number of means in the adapted GMMs equalled 120. Next, we evaluated the recognition accuracy for 3 cases: ( $i$ ) using all three states, (ii) using only the central state and (iii) using only the first and the third state. In the first case with all three states used the dimension of the super-vector constructed from the means of the GMM was 3,120. The length of the supervector is a product of 40 allophones $\times 3$ states $\times 26$ acoustic features $=3,120$. If we omitted the central state and only used the transition states the dimension of the super-vector lowered to 2,080, and finally if only the central state was used the dimension further reduced to 1,040.

In order to robustly evaluate the importance of different HMM states we conducted the same experiments as described above for the case of using a total of 200 Gaussians. To increase the number of Gaussians we split the distributions consisting of a single Gaussian in states that had the highest variance to the combination of Gaussians. Again, three tests were conducted using the above described combinations of states. The results of all experiments in emotion recognition using the FAU-Aibo corpus are shown in Table 2 In both cases of different number of Gaussians the results drop if only the central state is used however, if only the transition states are considered the result is slightly better. This observation leads us to believe that the first and the last state of the HMM capture more paralinguistic information than the central state of the monophone model.

The Vindat corpus transcript contains 41 unique allophones. The number is similar to the number of allophones in the FAU-Aibo corpus, hence the supervector dimensions are similar. The FAU-Aibo corpus has a defined set of training and test samples where as for the Vindat database an experimental protocol is not defined. Due to the smaller size of the corpus we conducted a 5-fold speaker-independent cross-validation. In each fold $20 \%$ of the samples was used for testing and $80 \%$ for training plus, the speakers from the test set were not included in the training set.

Table 3. Comparison of alcoholization recognition performance using different combinations of HMM states for the Vindat corpus

\begin{tabular}{c||c|c|c}
\multirow{2}{*}{\multicolumn{2}{c||}{ \# Gaussians }} & \multicolumn{3}{c}{ Number of used HMM states } \\
\cline { 2 - 4 } & States 1,2 and 3 & State 2 & States 1 and 3 \\
\hline 123 & $70.9 \%$ & $65.6 \%$ & $69 \%$ \\
200 & $69.2 \%$ & $64.4 \%$ & $67.6 \%$ \\
\hline
\end{tabular}


Similarly to the emotion recognition tests, we conducted experiments employing a different number of Gaussians. When only one Gaussian per state is used the total number of Gaussians equals 123. For the second evaluation the number of Gaussians was increased to 200. The results for the intoxication recognition are presented in Table 3. Opposite to the results in emotion recognition, the recognition rates for the alcoholization detection task are best if all HMM states are considered. The recognition accuracy drops in both attempts to omit some of the HMM states. Result is consistent regarding the complexity of the likelihood function, which is controlled by the number of Gaussians used.

\section{Conclusion}

We evaluated and assessed the importance of different HMM states in an HMM-based feature extraction method used to model paralinguistic information. Specifically, we evaluated the distribution of the paralinguistic information across different states of the HMM in two classification tasks: emotion recognition and alcoholization detection. In the task of recognizing emotions we found that the majority of emotion-related information is incorporated in the first and third state if standard 3-state HMMs are used. Surprisingly, in the alcoholization detection task we observed a somewhat equal distribution of information across all states, constantly producing better results if more states are utilized.

\section{References}

1. Schuller, B., Steidl, S., Batliner, A.: The INTERSPEECH 2009 Emotion Challenge. In: INTERSPEECH 2009. ISCA, pp. 312-315 (2009)

2. Schuller, B., Steidl, S., Batliner, A., Schiel, F., Krajewski, J.: The INTERSPEECH 2011 Speaker State Challenge. In: INTERSPEECH 2011. ISCA (2011)

3. Gajšek, R., Mihelič, F., Dobrišek, S.: Speaker state recognition using an hmm-based feature extraction method. Computer Speech and Language (to be published, 2012)

4. Steidl, S.: Automatic Classification of Emotion-Related User States in Spontaneous Children's Speech. Logos Verlag, Berlin (2009)

5. Mihelič, F., Gros, J., Dobrišek, S., Žibert, J.: Spoken language resources at luks of the university of ljubljana. International Journal of Speech Technology 6, 221-232 (2003)

6. Batliner, A., Steidl, S., Hacker, C., Nöth, E.: Private emotions vs. social interaction - a datadriven approach towards analysing emotion in speech. User Modeling and User-Adapted Interaction 18, 175-206 (2008)

7. Schiel, F., Heinrich, C., Barfüsser, S.: Alcohol language corpus: the first public corpus of alcoholized german speech. Language Resources and Evaluation (to appear, 2012)

8. Reynolds, D.A., Quatieri, T.F., Dunn, R.B.: Speaker Verification Using Adapted Gaussian Mixture Models. Digital Signal Processing 10, 19-41 (2000)

9. Young, S.J., Evermann, G., Gales, M.J.F., Hain, T., Kershaw, D., Moore, G., Odell, J., Ollason, D., Povey, D., Valtchev, V., Woodland, P.C.: The HTK Book, version 3.4.1. Cambridge University Engineering Department, Cambridge, UK (2009)

10. Hall, M., Frank, E., Holmes, G., Pfahringer, B., Reutemann, P., Witten, I.H.: The WEKA data mining software: an update. SIGKDD Explor. Newsl. 11, 10-18 (2009) 